\title{
Recurrent Left Colonic Diverticulitis Episodes: More Severe Than the Initial Diverticulitis?
}

\author{
Olivier Pittet · Nikos Kotzampassakis · \\ Sabine Schmidt • Alban Denys · Nicolas Demartines · \\ Jean-Marie Calmes
}

Published online: 16 January 2009

(c) Société Internationale de Chirurgie 2009

\begin{abstract}
Background Until recently, it was accepted that the rate of complications and failure of medical therapy were higher during recurrent episodes of diverticulitis. New data and new interpretation of older studies have challenged this opinion. The aim of the present study was to determine whether recurrent diverticulitis in comparison with the initial episode has a different short-term outcome after medical or surgical treatment.

Methods This was a retrospective cohort study of 271 consecutive patients admitted for diverticulitis confirmed by computed tomography (CT) between 2001 and 2004 . Altogether 202 patients had an initial episode (group I), and 69 had recurrent diverticulitis (group R). A total of 20 clinical and 15 radiologic parameters were analyzed and compared between the two groups, including need for surgery, clinical presentation at admission, response to treatment, complications, laboratory parameters, and pathologic CT features (colonic wall thickening, abscess, pneumoperitoneum, free intraperitoneal fluid). An unpaired Student's $t$-test and Fisher's and Wilcoxon's tests were applied for statistical analysis.

Results None of the clinical or radiologic parameters was statistically different between the two groups. Regarding surgery, $15.8 \%$ of the group I patients needed surgery at admission compared to $5.8 \%$ in group $\mathrm{R}(p=0.04)$.
\end{abstract}

O. Pittet · N. Kotzampassakis · N. Demartines $(\bowtie)$.

J.-M. Calmes

Department of Visceral Surgery, University Hospital CHUV,

Lausanne CH-1011, Switzerland

e-mail: demartines@chuv.ch

S. Schmidt · A. Denys

Department of Radiology, University Hospital CHUV,

Lausanne, Switzerland
Conservative treatment failure was similar in the two groups $(10.7 \%$ vs. $10.0 \%$; $p=0.84)$. There was $3 \%$ mortality at 30 days in group I compared to $0 \%$ in group R.

Conclusions Recurrent episodes of diverticulitis do not lead to more complications and more conservative treatment failure. Moreover, surgery at admission was less frequent among patients who presented with a recurrence.

\section{Introduction}

Until recently, it was accepted that the rate of complications and failure of medical therapy were higher during recurrent episodes of diverticulitis. New data and new interpretation of early studies have challenged this opinion. Diverticular disease is today a major health community problem, being the fifth most expensive among digestive diseases [1,2]. Acute diverticulitis is the most frequent complication and occurs in about $10 \%$ to $25 \%$ of the patients with diverticular disease [2-4]. The recurrence rate after a first episode of diverticulitis is estimated to be about $20 \%[4,5]$.

Results of diverticulitis management published recently are somewhat confusing [4, 6-14]. Outcomes after initial or recurrent diverticulitis episodes are described with different conclusions and various treatment options [4, 6-14]; and today's indications to elective surgery should be challenged as the current evidence is of little help. Based on the paucity of clear evidence, the American Society of Colon and Rectum Surgeons edited its guidelines in 2006 [13], and these recommendations about the management of recurrent diverticulitis remain open.

The aim of the present retrospective study was to determine whether recurrent diverticulitis has a different 
clinical and radiologic presentation and a different outcome from those parameters seen with a first episode.

\section{Methods}

Since 1998, all patients admitted to our hospital with a clinical suspicion of colonic diverticulitis underwent computed tomography (CT) scanning. Because of this policy, we were able to conduct a retrospective analysis of all patients admitted for diverticulitis between January 2001 and December 2004. After a computer review of all medical and radiologic reports, we identified all consecutive patients with a diagnosis of diverticulitis on their CT report at admission and those with a final diagnosis of diverticulitis on their discharge summary. Patients with a history of previous colonic surgery or coexisting colonic cancer were excluded. The local Institutional Ethic Committee approved the study.

Patients were divided into two groups: those with an initial episode of diverticulitis (group I) and those with a recurrence (group R). Recurrence was defined as a new episode of diverticulitis provided a previous CT scan confirmed the first episode. Patients with a diverticulitis diagnosis based on medical history only were excluded from the study.

\section{Clinical data}

Patient's medical reports were analyzed by two authors (O.P., N.K.) and were stored in a database to record their demographic, clinical, and laboratory parameters. Twenty clinical and biologic parameters were recorded-e.g., age, sex, co-morbidities, duration of symptoms, hospital stay, body temperature, leukocyte count, C-reactive protein (CRP) value at admission and during the first 48 hours, need for surgery, type of surgery, radiologic drainage, mortality and complications rates, antibiotic therapy, diet, readmission at 30 days). Hinchey's classification [15] was also recorded for patients who underwent surgery.

\section{Radiology workup}

A standardized abdominal multidetector CT (MDCT) protocol was performed within 24 hours after the patients' admission in the emergency department (LightSpeed; GE Healthcare, Milwaukee, WI, USA). A rectal enema with hydrosoluble iodinated contrast agent was applied followed by an intravenous injection (volume, in milliliters = body weight $+30 \mathrm{ml}$ ) of the iodinated contrast medium iohexol (Accupaque; Amersham Health, Wädenswil, Switzerland) at a flow rate of $3 \mathrm{ml} / \mathrm{s}$. Axial slices were then acquired during the venous phase (at $65 \mathrm{~s}$ ) from the diaphragm to the symphysis $(120 \mathrm{kV}, 200-240 \mathrm{~mA}$, table speed $15 \mathrm{~mm} /$ rotation, pitch 1.5 , collimation $4.0 \times 2.5 \mathrm{~mm}$, reconstructed thickness/interval $2.5 / 2.0 \mathrm{~mm}$ and $5 / 5 \mathrm{~mm}$, respectively). Iodinated contrast was not injected into patients with acute or chronic renal failure.

The CT images were retrospectively reviewed in consensus by two board-certified radiologists (S.S., A.D.), who checked 15 predetermined pathological CT features, mainly based on previous studies by Ambrosetti et al. [6, 16, 17]. These features included colonic wall thickening including its length, thickness, and localization; luminal narrowing; degree of diverticulosis; mesenteric fat stranding or edema including its extension; presence of free intraperitoneal fluid and localization; pericolonic abscess' size and localization; lymphadenopathy; presence and extension of extraluminal air or pneumoperitoneum; rectal contrast extravasation; and possible colovesical fistula.

Soft copy reading was done on a workstation with Advantage Windows (4.2 GE Healthcare).

\section{Therapeutic strategy}

Patients were initially treated with intravenous antibiotics according to institutional guidelines. They were amoxicillin and clavulanic acid (2.2 g three times daily IV followed by $625 \mathrm{mg}$ three times daily PO) or metronidazole (500 mg three times daily IV or PO) and ciprofloxacin (400 mg twice daily IV or $500 \mathrm{mg}$ twice daily PO). Antibiotic therapy was discontinued after 10 days according to institutional guidelines.

Surgery at admission was performed in severely ill patients with septic shock or clinical signs of general peritonitis associated with concordant radiologic findings. The staff in charge of the patient made the final decision for surgery.

Patients were considered to have conservative treatment if surgery or radiologic drainage were not performed during the first 24 hours. Conservative treatment failure was defined as the need for delayed surgery, radiologic drainage during hospitalization after the first 24 hours, or readmission or death during the 30 days following discharge. Delayed surgery was performed only in cases of clinical aggravation based on the above-mentioned criteria.

A subgroup analysis was also performed in group $\mathrm{R}$ comparing the patients with a second episode of diverticulitis to those with a third episode.

Due to sample size, independent statisticians performed a univariate analysis only. It consisted of the $\chi^{2}$ test and Fisher's exact test for qualitative variables analysis, the Mann-Whitney U-test for quantitative variable analysis, and the Wilcoxon test for nonparametric variable analysis. The threshold of significance was set at $p<0.05$. 


\section{Results}

A total of 271 consecutive patients met the inclusion criteria: 202 patients $(75 \%)$ with a first episode of diverticulitis (group I) and 69 patients (25\%) with a recurrent episode (group R). Among group R, 53 patients (77\%) had a second episode of diverticulitis, $13(19 \%)$ had a third episode, and $3(4 \%)$ had a fourth or fifth episode.

The two groups were similar regarding age and sex ratio, with a mean age of $61 \pm 14$ years in group I versus $62 \pm 13$ years in group $\mathrm{R}$ and percentages of $48 \%$ and $45 \%$ men, respectively. The presence of extraluminal gas bubbles of $>5 \mathrm{~mm}$ or pneumoperitoneum were observed in 52 patients $(25.7 \%)$ in group I versus 11 patients $(15.9 \%)$ in group $\mathrm{R}(p=0.14)$. Free intraperitoneal fluid was observed in 63 cases $(31.1 \%)$ in group I and 19 cases $(27.5 \%)$ in group $\mathrm{R}(p=0.65)$; and the mean CRP value was similar in the two groups $(125 \pm 91$ vs. $105 \pm 95 \mathrm{~g} / \mathrm{l}$, respectively; $p=0.14$ ). If we considered the criteria of Ambrosetti et al. for moderate and severe diverticulitis [6], there were $60(29.7 \%)$ patients with severe diverticulitis in group I compared to $15(21.7 \%)$ patients in group $\mathrm{R}$ $(p=0.21)$. Apart from the need for emergency surgery or radiologic drainage, which was more frequent in 32 patients with an initial episode of diverticulitis compared to 4 patients with recurrence $(15.8 \%$ vs. $5.8 \%)(p=0.04)$, there was no difference between groups concerning the other clinical, biologic, or radiologic parameters (Table 1).
In group I, 14 patients underwent a sigmoidectomy with primary end-to-end anastomosis, 13 had a sigmoidectomy with terminal colostomy (Hartmann procedure), and 5 had radiologic abscess drainage. In group $\mathrm{R}$, there were one sigmoidectomy with primary end-to-end anastomosis and three Hartmann procedures.

Conservative medical treatment was successful in 153 patients $(89.2 \%)$ in group I compared to 58 patients $(90.0 \%)$ in group $\mathrm{R}(p=0.84)$. The number of complicated cases, which included all patients who required surgery at admission or had conservative treatment failure, were similar in the two groups with 49 patients $(24.3 \%)$ in group I and 11 patients $(15.9 \%)$ in group $\mathrm{R}(p=0.18)$.

Delayed surgery due to conservative treatment failure was performed in nine patients $(5.3 \%)$ in group I compared to five patients $(7.8 \%)$ in group $\mathrm{R}(p=0.69)$. In group I, five had sigmoidectomy with primary anastomosis and four had Hartmann procedures. In group R, all five patients had sigmoidectomy with primary anastomosis. No radiologic drainage was needed after the time of admission in either group.

Regarding Hinchey's classification (Table 2) for emergency or delayed surgery, there was a trend for a higher proportion of patients with more severe diverticulitis (Hinchey III and IV) in group I, with 28 of 202 patients (13.9\%) compared to 3 of 69 patients $(4.3 \%)$ in group $\mathrm{R}$ $(p=0.06)$. The difference is even greater if we calculate the proportion of Hinchey III/IV among the operated

Table 1 Clinical and radiologic parameters

\begin{tabular}{|c|c|c|c|}
\hline Variable & First episode & Recurrence & $p$ \\
\hline Duration of symptoms (days) & 2.6 & 2.7 & 0.09 \\
\hline C-reactive protein at admission $(\mathrm{mg} / \mathrm{L})$ & 124.8 & 104.5 & 0.14 \\
\hline Leukocytes at admission (G/l) & 12.3 & 12.5 & 0.76 \\
\hline Temperature at admission $\left({ }^{\circ} \mathrm{C}\right)$ & 37.3 & 37.3 & 1 \\
\hline Obesity: $\mathrm{BMI}>35 \mathrm{~kg} / \mathrm{m}^{2}$ (no.) & $7(3.5 \%)$ & $6(8.7 \%)$ & 0.10 \\
\hline Hospital stay (days) & 8.6 & 9.7 & 0.64 \\
\hline Mortality (no.) & $6(3.2 \%)$ & 0 & 0.34 \\
\hline Modification of antibiotic therapy during hospital stay (no.) & $13(6.4 \%)$ & $5(7.4 \%)$ & 0.78 \\
\hline Length of colonic wall infiltration (mm) & 61 & 63 & 0.59 \\
\hline Thickness of colonic wall infiltration (mm) & 9 & 11 & 0.29 \\
\hline Mesosigmoid extension of edema (mm) & 59 & 61 & 0.41 \\
\hline Presence of abscess on CT scan (no.) & $32(15.8 \%)$ & $10(14.5 \%)$ & 0.85 \\
\hline Presence of intraperitoneal fluid on CT scan (no.) & $63(31.1 \%)$ & $19(27.5 \%)$ & 0.65 \\
\hline Distal sigmoid localization (no.) & $116(52.7 \%)$ & $34(49.3 \%)$ & 0.26 \\
\hline Extraluminal air bubble ( $>5 \mathrm{~mm}$ ) or pneumoperitoneum (no.) & $52(25.7 \%)$ & $11(15.9 \%)$ & 0.14 \\
\hline Emergency surgery or radiologic drainage (no.) & $32(15.8 \%)$ & $4(5.8 \%)$ & 0.04 \\
\hline
\end{tabular}

$B M I$ body mass index, $C T$ computed tomography

Apart from the need for emergency surgery or radiologic drainage, none of the other clinical or radiologic parameters analyzed were statistically different 
Table 2 Hinchey classification: number of patients in each group

\begin{tabular}{lll}
\hline & \multicolumn{2}{l}{ Number of patients } \\
\cline { 2 - 3 } Hinchey classification & First episode & Recurrence \\
\hline Surgery in emergency & 5 & 2 \\
$\quad$ Hinchey IV & 17 & 1 \\
Hinchey III & 5 & 1 \\
Hinchey II & & \\
Delayed surgery & 1 & 0 \\
Hinchey IV & 5 & 0 \\
Hinchey III & 2 & 5 \\
Hinchey II & 1 & 0 \\
Hinchey I & & \\
\hline
\end{tabular}

The higher ratio of patients with Hinchey III and IV in cases at the initial episode $(13.9 \%)$ compared to the recurrence episode $(4.3 \%)$ is nearly significant $(p=0.06)$

patients in each group, with 28 of $36(77.8 \%)$ in group I compared to 3 of 9 (33.3\%) patients in group $\mathrm{R}(p=0.01)$.

Mortality was zero patients in group $\mathrm{R}$ and six (3\%) patients in group I. The mean age of the deceased patients was significantly higher than the age of the surviving study population $(79 \pm 8$ vs. $61 \pm 14$ years $(p=0.002)$. Four patients died after emergency surgery; all had Hinchey IV peritonitis, and the reasons for death were related to their previous co-morbid health conditions (cancer, steroid, pneumonia, myocardial infarction). Two patients with concomitant noncolorectal oncologic disease refused emergency surgery and died of septic shock.

The other conservative treatment failure was readmission at 1 month in five patients $(2.5 \%)$ and two patients (2.9\%), respectively, in group I and group $\mathrm{R}(p=0.81)$. The overall length of hospital stay was similar in the two groups, with $9 \pm 7$ days and $10 \pm 10$ days $(p=0.64)$ in group I and group $\mathrm{R}$, respectively. Considering the successful conservative treatment only, the mean hospital stay was similar, with $8 \pm 6$ days in both groups.

The subgroup analysis comparing the patients with a second episode of diverticulitis to those admitted with a third episode showed that surgery at admission was performed in four patients $(7.5 \%)$ in the second-episode subgroup compared to none in the third-episode subgroup $(p=0.58)$. Complicated cases, as defined above, were also similar in these two groups with six patients $(11.3 \%)$ in the second-episode subgroup and two patients $(15.4 \%)$ in the third-episode subgroup $(p=0.37)$.

\section{Discussion}

This study suggests with sufficient statistical power that recurrent episodes of diverticulitis have no increased risk of conservative treatment failure or complications compared to what occurs during the initial episode. Successful conservative treatment was similar for the initial episode and recurrence; and it is of note that no mortality was related to recurrence. Clinical and radiologic presentations were similar. Patients with recurrent diverticulitis had even less risk of needing emergency surgery. Hinchey III and IV diverticulitis were less frequently observed with recurrent diverticulitis.

Our findings confirm that the number of attacks of diverticulitis is no longer an overriding factor for elective surgery. Early studies and guidelines [4, 14, 18], based principally on inappropriate interpretation of pioneer works by Parks [19], as explained by Janes et al. [9], should definitely be left behind.

None of the 20 clinical and radiologic parameters analyzed were statistically different, and patients with recurrent episodes did not come earlier to hospital after the onset of symptoms despite the fact that they already had an episode of diverticulitis in the past.

The efficiency of conservative treatment and the response to medical treatment based on the length of hospital stay were similar in the two groups. Thus, recurrent diverticulitis should no longer be considered a risk factor for conservative treatment failure. Our study shows a significant threefold higher rate of a need for emergency surgery among patients with initial cases of diverticulitis than among those with a recurrence. Considering only the 36 patients who needed emergency surgery, $89 \%$ had no history of a previous episode of acute diverticulitis. This higher proportion of emergency surgery required for initial diverticulitis has also been described by other authors. Lorimer [10] and Nylamo [12] reported in 154 and 48 patients, respectively, that more than $90 \%$ of their patients who underwent emergency operations for complicated diverticulitis had no history of a previous episode of acute diverticulitis. This was also pointed out by Chapman et al. [7], who found in a cohort study of 330 patients with complicated diverticular disease that $68 \%$ of the patients with a perforation had their first episode of diverticulitis. Haglund et al., in a study of 392 patients with diverticulitis followed over a 12 -year period, noted that only $25 \%$ had a recurrence. Among these patients, none had a perforation; and medical management was sufficient in all cases [20].

The comparison of Hinchey grade in the initial and recurrent episodes of diverticulitis showed that complicated diverticulitis, defined as Hinchey III or IV, was associated with initial episodes of diverticulitis in most cases. The reason of this difference is not clear, but one hypothesis is that local factors, such as adhesion status due to previous diverticulitis or/and a large bowel fibrous scar, may prevent extension of the infection process within the peritoneum during recurrences. We could even hypothesize 
that recurrence may have a protective role regarding perforation or local complications of diverticulitis, but a larger data pool is needed confirm this hypothesis.

Mortality was observed only during initial episodes, but the important point is that no patient with conservative treatment died during follow-up. In the literature, mortality seems to be limited to the high-risk aged population with multiple co-morbidities [21] who require emergency surgery. Because this population is at higher risk of mortality in case of a new episode, elective sigmoid resection should probably be proposed after an appropriate preoperative evaluation and stabilisation of their risk factors.

Subsequent episodes of diverticulitis do not represent a risk factor for poor outcomes, as the subgroup analysis of patients with two episodes of diverticulitis compared to those with three episodes did not reveal any difference in terms of morbidity or need for surgery. This fact was also pointed out by Chapman et al. [8], who found a lower perforation rate and equivalent morbidity and mortality rates in the case of multiple diverticulitis episodes compared to one or two previous episodes only.

A limitation of the present study is its retrospective design. However, the analyzed population is homogeneous and consecutive over 4 years, and the diagnosis and treatment strategy were standardized and defined prior to beginning the study. Another possible bias is the sample size, which did not allow multivariate analysis. However, the number of patients is comparable to that in other studies dealing with diverticulitis recurrence. The 2006 guidelines of the American Society of Colon and Rectum Surgeons are based on the study by Mueller et al. [11] and Chapman et al. [7], who dealt with long-term follow-up of, respectively, 360 and 330 patients with diverticulitis. Moreover, in the mentioned studies, there is little information about clinical and radiologic differences between initial and recurrent episodes of diverticulitis.

The fact that the indication for surgery was decided on by the staff in charge, including several surgeons, may be regarded as a selection bias in our study. however, the indications were adequate, as most cases were found to be Hinchey III and IV during the operation. The cases for whom the indication for surgery remain questionable are the few cases of Hinchey I and II encountered more frequently in recurrence group. They might be regarded as overtreatment. However those cases do not change the fact that the initial episode of diverticulitis is generally associated with a higher rate of surgical cases.

A discussion point of our present study is that we did not stratify the severity of the initial episode in our analysis because of the lack of a validated diverticulitis severity score in the literature-except the CT-based criteria of Ambrosetti et al. [6]. Their study demonstrated that initial severe episodes of diverticulitis have a higher risk for severe recurrent episodes (36\%) compared to moderate initial episodes (17\%). Thus, patients with an initial severe episode had a moderate recurrence episode in $64 \%$ of cases. This suggests that a severe initial episode of diverticulitis is probably a risk factor for a severe recurrent episode but with a low positive predictive value, which should be further investigated.

\section{Conclusions}

Our study shows that recurrent diverticulitis is not associated more often with conservative treatment failure or poor outcomes than an initial episode. Patients with recurrent diverticulitis may be treated conservatively without complication risks.

\section{References}

1. Painter NS, Burkitt DP (1975) Diverticular disease of the colon, a 20th century problem. Clin Gastroenterol 4:3-21

2. Schoetz DJ Jr (1999) Diverticular disease of the colon: a centuryold problem. Dis Colon Rectum 42:703-709

3. Parks TG (1975) Natural history of diverticular disease of the colon. Clin Gastroenterol 4:53-69

4. Stollman NH, Raskin JB (1999) Diagnosis and management of diverticular disease of the colon in adults; Ad Hoc Practice Parameters Committee of the American College of Gastroenterology. Am J Gastroenterol 94:3110-3121

5. Kaiser AM, Jiang JK, Lake JP et al (2005) The management of complicated diverticulitis and the role of computed tomography. Am J Gastroenterol 100:910-917

6. Ambrosetti P, Becker C, Terrier F et al (2002) Colonic diverticulitis: impact of imaging on surgical management: a prospective study of 542 patients. Eur Radiol 12:1145-1149

7. Chapman J, Davies M, Wolff B et al (2005) Complicated diverticulitis: is it time to rethink the rules? Ann Surg 242:576-581

8. Chapman JR, Dozois EJ, Wolff BG et al (2006) Diverticulitis: a progressive disease? Do multiple recurrences predict less favorable outcomes? Ann Surg 243(6):876-883

9. Janes S, Meagher A, Frizelle FA (2005) Elective surgery after acute diverticulitis. Br J Surg 92:133-142

10. Lorimer JW (1997) Is prophylactic resection valid as an indication for elective surgery in diverticular disease? Can J Surg 40:445-448

11. Mueller MH, Glatzle J, Kasparek MS et al (2005) Long-term outcome of conservative treatment in patients with diverticulitis of the sigmoid colon. Eur J Gastroenterol Hepatol 17:649-654

12. Nylamo E (1990) Diverticulitis of the colon: role of surgery in preventing complications. Ann Chir Gynaecol 79:139-142

13. Rafferty J, Shellito P, Hyman NH et al (2006) Practice parameters for sigmoid diverticulitis. Dis Colon Rectum 49:939-944

14. Wong WD, Wexner SD, Lowry A et al (2000) Practice parameters for the treatment of sigmoid diverticulitis: supporting documentation; The Standards Task Force, The American Society of Colon and Rectal Surgeons. Dis Colon Rectum 43:290-297

15. Hinchey EJ, Schaal PG, Richards GK (1978) Treatment of perforated diverticular disease of the colon. Adv Surg 12:85-109

16. Ambrosetti P, Robert J, Witzig JA et al (1992) Prognostic factors from computed tomography in acute left colonic diverticulitis. $\mathrm{Br}$ J Surg 79:117-119 
17. Ambrosetti P, Jenny A, Becker C et al (2000) Acute left colonic diverticulitis - compared performance of computed tomography and water-soluble contrast enema: prospective evaluation of 420 patients. Dis Colon Rectum 43:1363-1367

18. Kohler L, Sauerland S, Neugebauer E (1999) Diagnosis and treatment of diverticular disease: results of a consensus development conference; The Scientific Committee of the European Association for Endoscopic Surgery. Surg Endosc 13:430-436

19. Parks TG (1969) Natural history of diverticular disease of the colon: a review of 521 cases. BMJ 4:639-642
20. Haglund U, Hellberg R, Johnsen C et al (1979) Complicated diverticular disease of the sigmoid colon: an analysis of short and long term outcome in 392 patients. Ann Chir Gynaecol 68:41-46

21. Pessaux P, Muscari F, Ouellet JF et al (2004) Risk factors for mortality and morbidity after elective sigmoid resection for diverticulitis: prospective multicenter multivariate analysis of 582 patients. World J Surg 28:92-96 